\title{
Recognition of Operator DNA by Tet Repressor
}

\author{
Christian Berens ${ }^{\dagger}$ and Dietmar Porschke* ${ }^{\ddagger}$ \\ ${ }^{\dagger}$ Department Biologie, Universität Erlangen-Nürnberg, 91058 Erlangen, Germany \\ ${ }^{\ddagger}$ Max Planck Institut für biophysikalische Chemie, 37077 Göttingen, Germany
}

ABSTRACT: The recognition of operator DNA by Tet repressor was analyzed by fluorescence stopped flow measurements. The main part of the fluorescence change observed for the reaction of the repressor with operator DNA reflects a second-order binding reaction including the expected concentration dependence. Global fitting of transients measured at different concentrations reveal at least one intramolecular step in addition to the bimolecular step. The rate constant for the bimolecular step is strongly salt dependent approaching the limit of diffusion control $2 \times 10^{8} \mathrm{M}^{-1} \mathrm{~s}^{-1}$ at $50 \mathrm{mM} \mathrm{NaCl}$ and

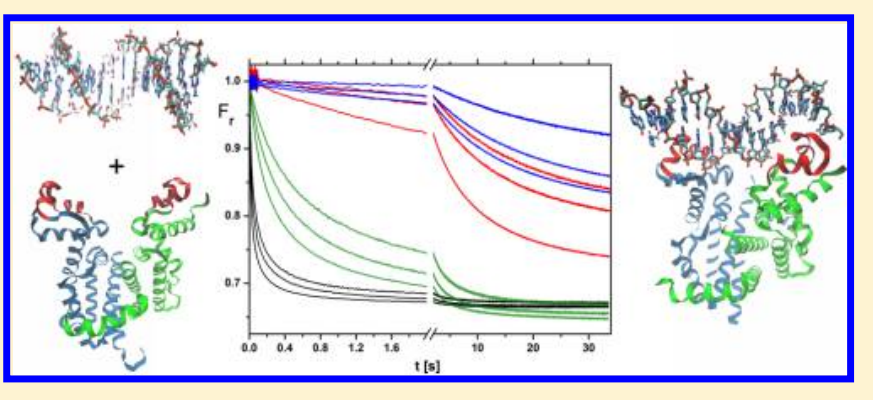
decreasing to $5 \times 10^{4} \mathrm{M}^{-1} \mathrm{~s}^{-1}$ at $600 \mathrm{mM} \mathrm{NaCl}$. These data are consistent with initial formation of a pre-equilibrium complex; electrostatic steering resulting from the high dipole moment of the repressor may contribute to the strong salt dependence. The rate constants of the intramolecular step are in the range of $\sim 0.1 \mathrm{~s}^{-1}$. Fluorescence quenching is salt dependent; the overall binding constant to operator $\mathrm{O} 1$ at $150 \mathrm{mM} \mathrm{NaCl}$ is $5 \times 10^{8} \mathrm{M}^{-1}$; binding constants at different salt concentrations indicate $\sim 5$ ion contacts for the specific complex of the Tet repressor dimer. The binding constant to operator $\mathrm{O} 2$ is higher than to $\mathrm{O} 1$ by a factor of $\sim 2$ at $400 \mathrm{mM} \mathrm{NaCl}$.

\section{INTRODUCTION}

The assignment of quantitative parameters for specific interactions between proteins and nucleic acids is not always simple. A prominent example is the interaction of lac repressor with its operator - critical parameters had to be analyzed by filter binding methods ${ }^{1,2}$ and discussions about the validity and implications of these parameters are still going on. ${ }^{3-5}$ The Tet repressor (TetR) seemed to be much more convenient in this respect because binding to its operator is associated with a considerable decrease of its fluorescence. Nevertheless, there are contradictory conclusions ${ }^{6,7}$ on the kinetics of operator recognition in the literature. The first analysis indicated a rather simple reaction with an initial pre-equilibrium followed by relatively fast recognition of the operator sequence. ${ }^{6}$ The results of a subsequent investigation are quite different ${ }^{7}-$ the authors used much higher reactant concentrations and applied quasifirst-order conditions with a large excess of the operator DNA - the resulting association rate constants are relatively low and also do not show the strong salt dependence detected in the first investigation. There are many differences in the details of the analysis including the use of natural restriction fragments versus short synthetic DNA fragments, of wild-type Tet repressor versus a mutant repressor with a single Trp residue at position 43 and a simple form to consider the existence of a photoreaction versus disregard of this perturbation. The reaction is now analyzed in more detail than previously. Part of the problem is selection of an operator DNA without complications resulting from a duplex-hairpin transition of the palindromic sequence. Another problem is correction of data for the effects of a photoreaction. Using carefully selected reactants and a ratio procedure for correction of the photoreaction effects, the conclusions of Kleinschmidt et al. ${ }^{6}$ on the rates of the bimolecular step are confirmed, but some other previous conclusions have to be modified.

\section{MATERIALS AND METHODS}

The Tet repressor from transposon Tn10, which belongs to class $\mathrm{B},{ }^{8}$ was prepared by using an overproducing strain as described. $^{9}$ The repressor concentration was determined spectrophotometrically using an extinction coefficient of 30.6 $\times 10^{3} \mathrm{~cm}^{2} / \mathrm{mol}$ at $280 \mathrm{~nm}$ for the dimer. ${ }^{10}$

The DNA fragments were prepared by phosphoramidite synthesis and purified by reversed phase HPLC (IBA, Göttingen). Extinction coefficients of DNA fragments were evaluated based on predictions of their secondary structure using the routines of the Vienna package ${ }^{11}$ and routines for calculation of extinctions coefficients for given sequences in their predicted secondary structure. ${ }^{12,13}$ The following fragments were used

o1_a: ACTCTATCATTGATAGAGTT; o1_b: AACTC TATCAATGATAGAGT; $01 \_l: \quad$ CGA CACTCTATCATTGATAGAGTCTTTTTGACTCTATCAATGATAGAGTGTCG; o2 $l$ : CCACTCCCTATCAGTGATAGAGAAAAGCTTTTTGCTTTTCTCTATCACTGATAGGGAGTGG.

Received: December 3, 2012

Revised: January 14, 2013

Published: January 17, 2013 
Solutions for stopped flow measurements were made up in a standard buffer containing $10 \mathrm{mM}$ Tris $\mathrm{pH}$ 8.0, 0.1 mM EDTA, $0.1 \mathrm{mM}$ DTT together with specified concentrations of $\mathrm{NaCl}$.

The kinetics was recorded by a home-built stopped-flow instrument. A Hamamatsu $200 \mathrm{~W}$ mercury-xenon lamp together with a Bausch\&Lomb high intensity grating monochromator was used for excitation at $296.7 \mathrm{~nm}$. The photoelectric signal was digitized by a Spectrum PCI.212 with 12 bit amplitude resolution and a $128 \mathrm{M}$-sample storage (Spectrum, 22927 Grosshansdorf, Germany). The transients were sampled at $500 \mathrm{kHz}$ or $2 \mathrm{MHz}$ depending on the required time range. Extensive condensation of data, in particular at long times, was applied for optimal noise ratios of each individual transient. This is the reason for the increase in the signal-tonoise ratio at long times. The signal-to-noise ratio was also increased by averaging of up to 20 transients. The averaged reaction transients were corrected for photoeffects by a ratio procedure used previously ${ }^{14}$ and described now in more detail in the section: Processing of fluorescence stopped flow data.

Molecular graphics was designed with $\mathrm{VMD}^{15}$ the structures of Protein Data Bank entries 1a61 ${ }^{16}$ and 1qpi ${ }^{17}$ together with a DNA structure generated with NAMOT ${ }^{18}$ were used.

\section{RESULTS}

Hairpin-Duplex Transition of Operator DNA. The DNA fragments o1_a and o1_b are complementary strands of the Tet-operator O1. Annealed 1/1 mixtures were used by Kedracka-Krok et al. ${ }^{7}$ for their experiments on TetR binding to operator DNA. o1_a and o1_b have a palindromic sequence and thus $1 / 1$ mixtures may exist as duplex or as hairpin structure. Because duplexes have more base pairs, their stability may be expected to be higher than that of hairpins. However, duplex formation requires association of two strands. Thus, the equilibrium between the duplex and hairpin forms is concentration dependent and hairpins are favored at low concentrations. This general expectation was confirmed by studies on equimolar mixtures of o1_a and o1_b. When this pair of strands is annealed at a high concentration, a dominant population of duplexes is expected, whereas hairpins should be favored by annealing at low concentrations. Measurements of the absorbance at $260 \mathrm{~nm}$ show that the transition between the forms is slow at room temperature (time constants in the range of $24 \mathrm{~h}$ at oligomer concentrations of 0.1 to $0.4 \mu \mathrm{M}$ at $20^{\circ} \mathrm{C}$ ). Thus, both forms can be considered as metastable on the time scale of experiments at low temperatures.

The relevance of the hairpin-duplex transition for binding of Tet repressor was demonstrated by a simple stopped flow analysis: Tet repressor was mixed with $1 / 1$ mixtures of o1_a + ol b of identical composition but prepared under different annealing conditions. As shown in Figure 1, the fluorescence transients are strongly dependent on these conditions. A particularly low fluorescence change is observed for a sample cooled quickly from the denatured state at the final, low DNA concentration; slow annealing at the same low DNA concentration leads to a clearly higher fluorescence change; the fluorescence change is increased further after annealing at a high DNA concentration, before the sample was diluted to the final concentration used for the stopped flow measurements; finally, a small additional increase of the fluorescence change was observed; when the sample annealed at high DNA concentration was kept after dilution to the final state at 2 ${ }^{\circ} \mathrm{C}$ for $20 \mathrm{~h}$. As expected from the different annealing

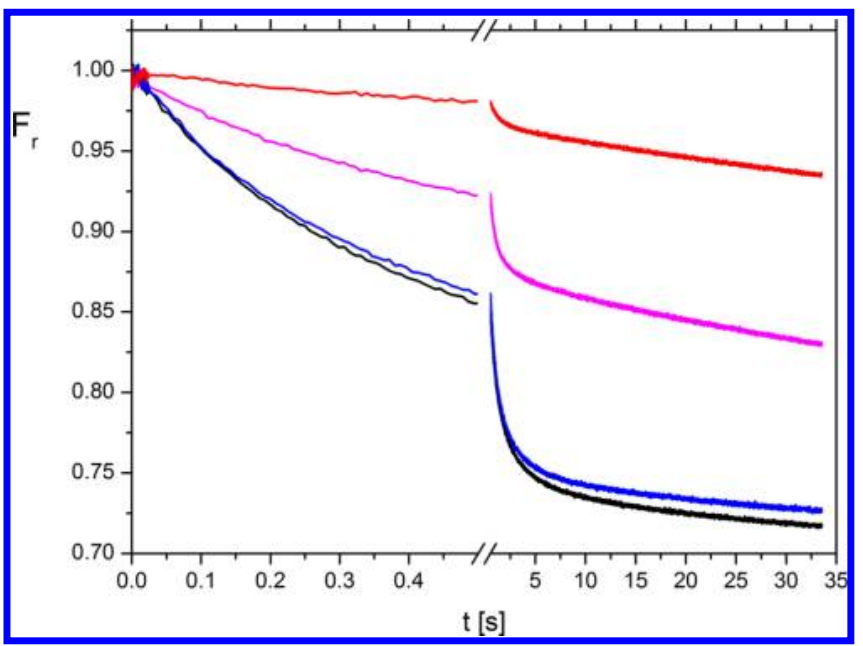

Figure 1. Fluorescence transients measured upon 1/1 stopped flow mixing of $0.3 \mu \mathrm{M}$ Tet repressor with $0.356 \mu \mathrm{M}$ DNA in standard buffer containing $150 \mathrm{mM} \mathrm{NaCl}$ without correction for photobleaching. The DNA samples were 1/1 mixtures of o1 a and o1 b annealed under different conditions: (a) quickly cooled at the final, low DNA concentration (orange); (b) slowly annealed at the final, low DNA concentration (red); (c) slowly annealed at $45.6 \mu \mathrm{M}$ DNA concentration in $30 \mathrm{mM}$ salt $\mathrm{pH} 7$ before transfer into the final buffer at $0.356 \mu \mathrm{M}$ DNA (blue); (d) as (c) but left in the final buffer/DNA concentration $30 \mathrm{~h}$ in a refrigerator at $2{ }^{\circ} \mathrm{C}$ (black). The fluorescence intensity $F_{\mathrm{r}}$ of the transients is normalized to 1 at $t=0$.

conditions, the fluorescence change increases with an increasing population of duplexes.

Another complication comes from the fact that the state of samples may be changed by rearrangements during storage and/or handling. Although these rearrangements are slow under typical experimental conditions, it is not trivial to exclude them. Calculations based on RNAcofold ${ }^{11}$ using DNA parameters indicate that the duplex population formed in $1 / 1$ mixtures of o1_a and o1_b at a concentration of $0.1 \mu \mathrm{M}$ at equilibrium corresponds to $28 \%$ at $25{ }^{\circ} \mathrm{C}-$ most of the DNA strands are in the hairpin form.

Finally, electrophoresis (in a $20 \%$ polyacrylamide gel) of a sample with a 1/1 mixture of o1_a and o1_b, slowly annealed at high DNA concentration, showed a hairpin population of $\sim 40 \%$. In summary, preparation of DNA samples with a dominant duplex population should be possible but requires careful selection of conditions during preparation and processing of the samples.

These complications can be avoided by using DNA constructs, which do not convert between different structures. For this purpose the + and - strands of the Tet-operator were included in a single sequence with a short connection sequence o1 $l$ (cf. Materials and Methods), such that these strands can form the operator duplex via a short connecting loop. Calculations based on RNAcofold ${ }^{11}$ using DNA parameters predict that both o1 $l$ and $02 \_l$ are in the expected hairpin form under the experimental conditions used in the present investigation and do not form dimers.

Processing of Fluorescence Stopped Flow Data. The analysis of the experimental data requires (1) correct scaling of transients measured at different reactant concentrations and (2) correction for a photobleaching effect. For optimal resolution of the kinetic data, fluorescence transients were recorded as difference signals using individual amplifications at different reactant concentrations. A signal reflecting the overall 
fluorescence intensity is obtained by addition of the preset offset signal, which is corrected by subtraction of a background signal. The background signal is mainly due to scattering effects and is measured at given instrument settings using the stopped flow cell filled with buffer. The background signal is read directly after measurement of the reaction transients to ensure the same state of light flux from the arc lamp.

All of the fluorescence signals are affected by photobleaching. Tests indicated that under the present experimental conditions the degree of bleaching is similar for the free and the DNAbound state of the protein. For correction of individual reaction transients, photobleaching was recorded for the same DNAprotein mixtures but equilibrated before subjection to the stopped flow. The photobleach signal was recorded directly after measurement of reaction transients to ensure the same state of light flux from the arc lamp. The background-corrected bleach signal was then normalized to 1.0 at time 0 . Division of the background-corrected reaction transients by the background-corrected and normalized bleach signal provided corrected reaction transients.

The magnitude of the photobleach correction was in the range of $\sim 3 \%$ after excitation for $135 \mathrm{~s}$ under standard conditions. Measurements at different excitation intensities involving a change of the light intensity by a factor of 2.5 provided transients, which were identical after application of the correction procedure within experimental accuracy.

Reaction Transients and Their Evaluation. Fluorescence transients for the reaction of Tet repressor with operator DNA in the form of o1 $l$ were recorded at 4 different salt concentrations. The transients were measured for $\sim 1 / 1$ mixtures of the reactants - with a slight excess of DNA. Under these conditions, the reaction appears in a convenient time range and the fluorescence change is relatively large. The main features of the reaction are illustrated by sets of corrected reaction transients at different salt concentrations with three different reactant concentrations each (Figure 2). For a simple, direct comparison, the fluorescence intensity $F_{\mathrm{r}}$ at $t=0$ of all transients was normalized to 1 . The time required for approach of the equilibrium state decreases continuously with decreasing salt concentration indicating increasing rates of reaction with decreasing salt. The dependence of the transients on the reactant concentration at each different salt concentration is clearly visible in all cases reflecting the dominant second-order term of the reaction. Finally, the dependence of the fluorescence quenching at the end of equilibration on the reactant concentration indicates the different levels of equilibrium constants.

For an optimal quantitative evaluation, it does not make sense to fit transients separately. An essential part of the information is given by the change of the transients with the reactant concentration as described above. Thus, global fitting is required and fitting should not involve more parameters than necessary. For this goal, the transients must be scaled such that a single set of optical parameters can be used: the fluorescence intensity $F_{\mathrm{p}}$ of the transients is scaled at $t=0$ according to the initial protein concentration. For a given set of transients, the initial level of the transient with the lowest protein concentration $c_{\mathrm{p}}{ }^{l}$ was set to 1.0, whereas the initial level of transients at higher protein concentrations $c_{\mathrm{p}}{ }^{\mathrm{h}}$ was set to $c_{\mathrm{p}}{ }^{\mathrm{h}} / c_{\mathrm{p}}{ }^{l}$.

All of the sets of transients (Figure 2) show a clear dependence on the reactant concentration expected for a bimolecular reaction, but a simple bimolecular reaction

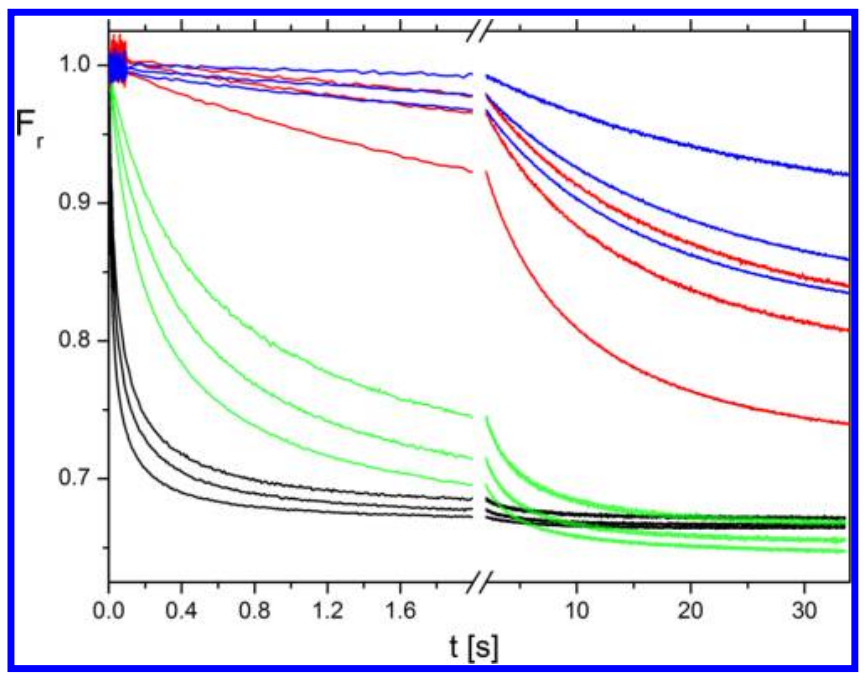

Figure 2. Corrected fluorescence transients measured for the reaction of Tet repressor with o1 $l$ operator DNA in standard buffer with 50, 150,400 , and $600 \mathrm{mM} \mathrm{NaCl}$ (black, green, red, and blue lines, respectively) at $20{ }^{\circ} \mathrm{C}$. For each salt concentration there are 3 transients with different reactant concentrations: $0.1,0.15$, and $0.3 \mu \mathrm{M}$ Tet repressor with $0.1105,0.1656$, and $0.331 \mu \mathrm{M}$ o1_l operator DNA respectively at 50,150 , and $400 \mathrm{mM} \mathrm{NaCl} ; 0.15,0.3$, and $0.4 \mu \mathrm{M}$ Tet repressor with $0.1655,0.331$, and $0.414 \mu \mathrm{M}$ o1_l operator DNA respectively at $600 \mathrm{mM} \mathrm{NaCl}$. In all cases, the rate of the approach to equilibrium follows the expected dependence on the reactant concentration. The fluorescence intensity $F_{\mathrm{r}}$ of the transients is normalized to 1 at $t=0$.

mechanism is not sufficient for fitting. The experimental data indicate that there is at least one additional reaction step. Global fitting by DynaFit ${ }^{19,20}$ demonstrated that the following mechanism

$$
A+B \underset{k_{1}}{\stackrel{k^{+}}{\rightleftarrows}} C \underset{k_{2}}{\stackrel{k_{2}^{+}}{\rightleftarrows}} D
$$

provides a satisfactory description of the experimental data. In addition to the four rate constants specified in eq 1 , fitting requires optical parameters for the three species contributing to fluorescence emission resulting in a total of seven fit parameters. Because the fluorescence intensity at $t=0$ is normalized (above), the optical parameter for the free protein is fixed and, thus, six fit parameters remain. Sets of three reaction transients recorded at different concentrations proved to be sufficient to define these parameters at a reasonable accuracy (cf. Figure 3).

Minor deviations of the fit from the experimental data are due to different reasons. First of all, three different transients measured separately are combined and transformed to a common scale. Transformation is executed as carefully as possible (cf. description above), but some deviations due to experimental errors cannot be avoided. Obviously, the quality of fits can be improved by addition of another reaction step or by using individual optical parameters for each transient. However, at the given state of the experimental data an extension in the number of fit parameters does not seem to be reasonable.

The data recorded at the other salt concentrations (Figure 2) were analyzed by the same procedure. The resulting parameters are combined in Table 1 . The rate constants $k_{1}^{+}, k_{1}^{-}, k_{2}^{+}$, and $k_{2}^{-}$ are defined in eq 1 . The equilibrium constants are given by $K_{1}=$ 


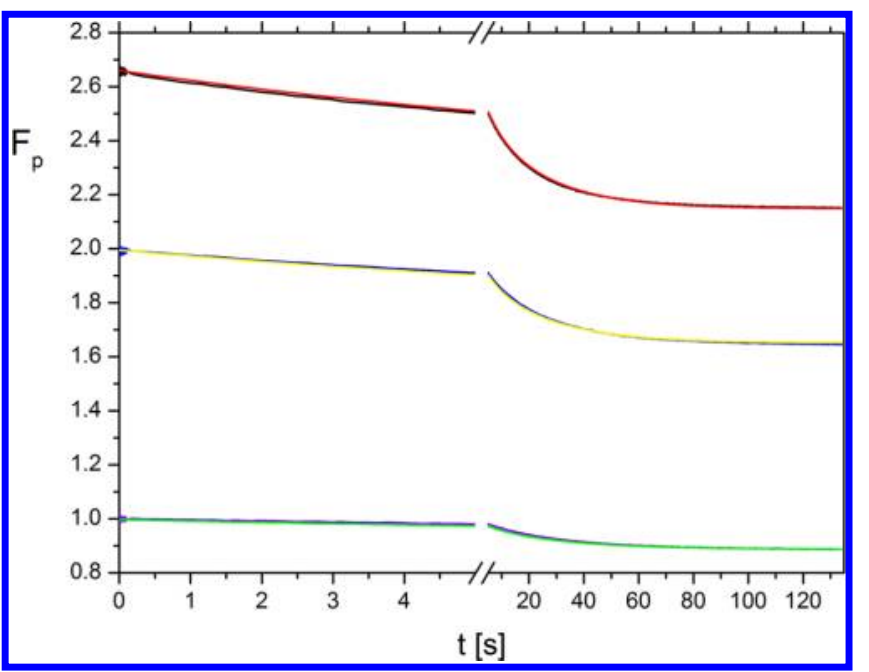

Figure 3. Global fit of transients measured for the reaction of Tet repressor with o1 $l$ operator DNA at $600 \mathrm{mM} \mathrm{NaCl}$ at different reactant concentrations: $0.15+0.1655 \mu \mathrm{M}$ (violet/green), $0.3+0.331$ $\mu \mathrm{M}$ (blue/yellow), and $0.4+0.414 \mu \mathrm{M}$ (black/red); the first and second value represent the concentration of Tet repressor and operator DNA, respectively; the first and the second color represent the experimental data and the fit, respectively. The fluorescence intensity $F_{\mathrm{p}}$ is normalized at $t=0$ according to the total Tet repressor concentration, as described in the text. The fitted parameters are given in Table 1.

$k_{1}^{+} / k_{1}^{-}, K_{2}=k_{2}^{+} / k_{2}^{-}$and $K=K_{1} *\left(K_{2}+1\right)$. The parameter 'quench' gives the fluorescence intensity of the states $C$ and $D$ at equilibrium with respect to that of the free protein. The individual quenching parameters of the states $C$ and $D$ are not given because they could not be determined at a sufficient accuracy. The error of $k_{1}^{+}$presented by DynaFit is $\pm 1 \%$, but the accuracy is estimated to be $\pm 10 \%$. The error presented by DynaFit for the other rate constants is in the range of 5 to $10 \%$; their accuracy is estimated to be $\pm 25 \%$. A major source of information for the magnitude of the overall binding constant $K$ is the concentration dependence of quenching approached at $t$ $=\infty$. Fits of these quenching parameters by an independent fitting program based on the simple law of mass action provided $K$-values close to those obtained by DynaFit. The accuracy of $K$-values in the range of 150 to $600 \mathrm{mM} \mathrm{NaCl}$ is estimated to be $\pm 15 \%$.

A very strong dependence on the salt concentration is found for the forward rate of the first step and consequently for the binding constants $K$ and $K_{1}$. In comparison to these large effects, the salt dependence of the other parameters including the equilibrium quenching is relatively small. However, the salt dependence of quenching has clear implications on the evaluation and the magnitude of the equilibrium constants.

Plots of the logarithm of the overall binding constants as a function of the logarithm of the ion concentration demonstrate that the value obtained at $50 \mathrm{mM} \mathrm{NaCl}$ is lower than expected from the linear dependence observed for the other values. Part of this deviation seems to be due to the experimental conditions, which are unfavorable for measuring the very large binding constant at $50 \mathrm{mM} \mathrm{NaCl}$. We note that this problem at $50 \mathrm{mM} \mathrm{NaCl}$ does not affect the accuracy of the $k_{1}^{+}$value, but mainly results from a limited accuracy of the $k_{1}^{-}$-value. The salt dependence ${ }^{21,22}$ of $K$ observed in the range from 150 to $600 \mathrm{mM} \mathrm{NaCl}$ indicates a value of $\sim 5$ ion contacts in the repressor-operator complex.

The results obtained for binding of the wt Tet repressor to the operator o1 $l$ have been supplemented by a set of data obtained for binding to the operator o 2 at $400 \mathrm{mM} \mathrm{NaCl} \mathrm{pH}$ 8. The parameters are similar, but the overall binding constant to operator $02 \_l$ is higher by a factor of $\sim 2$ than that to o1 $l$, which is consistent with previous measurements of dissociation rates using the filter binding technique. ${ }^{23,24}$ Another set of data for the binding of wt Tet repressor to o1_l was obtained at 600 $\mathrm{mM} \mathrm{NaCl} \mathrm{pH} 7$ indicating that the change of parameters remains relatively small upon $\mathrm{pH}$ variation in this range.

Finally, two Tet repressor mutants were compared qualitatively. A mutant with a replacement of Trp at position 75 by Phe showed a stopped flow transient at $150 \mathrm{mM} \mathrm{NaCl}$ $\mathrm{pH} 8$ similar to that recorded for the wt-repressor under equivalent conditions. The fluorescence quenching observed at the reactant concentration used in this experiment was higher for the mutant than that found in a corresponding experiment with the wt-repressor. A detailed assignment of differences requires a more extensive analysis. A mutant with a replacement of Trp at position 43 by Tyr revealed a completely different transient upon reaction with operator ol $l$ at $150 \mathrm{mM} \mathrm{NaCl}$ $\mathrm{pH} 8$ : a slow increase of the fluorescence by $\sim 3.5 \%$ with time constants of $\sim 20 \mathrm{~s}$ was observed - the large, fast component characteristic of the wt-repressor did not appear.

\section{DISCUSSION}

The striking difference between the results of Kleinschmidt et al. ${ }^{6}$ and of Kedracka-Krok et al. ${ }^{7}$ was the starting point for the present investigation. Binding of Tet repressor to operatorDNA can be analyzed more conveniently than other DNA binding reactions because of a relatively large fluorescence change and thus it should be possible to characterize the reaction without ambiguity in its basic features. The first investigation by Kleinschmidt et al. ${ }^{6}$ was limited to low reactant concentrations because operator DNA in the form of restriction fragments was not available for measurements at high

Table 1. Parameters Obtained from Global Fitting of Reaction Transients (Definitions Eq 1 and Text) ${ }^{a}$

\begin{tabular}{|c|c|c|c|c|c|c|c|c|c|c|}
\hline DNA & $\mathrm{pH}$ & $\mathrm{NaCl}$ & $k_{1}^{+}$ & $k_{1}^{-}$ & $k_{2}^{+}$ & $k_{2}^{-}$ & $K_{1}$ & $K_{2}$ & K & quench \\
\hline & & {$[\mathrm{mM}]$} & {$\left[\mathrm{M}^{-1} \mathrm{~s}^{-1}\right]$} & {$\left[\mathrm{s}^{-1}\right]$} & {$\left[\mathrm{s}^{-1}\right]$} & {$\left[\mathrm{s}^{-1}\right]$} & {$\left[\mathrm{M}^{-1}\right]$} & & {$\left[\mathrm{M}^{-1}\right]$} & \\
\hline ol & 8 & 50 & $2.03 \times 10^{08}$ & 0.110 & 0.140 & 0.743 & $1.85 \times 10^{09}$ & 0.19 & $2.20 \times 10^{09}$ & 0.657 \\
\hline ol & 8 & 150 & $1.55 \times 10^{07}$ & 0.039 & 0.037 & 0.138 & $3.94 \times 10^{08}$ & 0.27 & $4.98 \times 10^{08}$ & 0.632 \\
\hline o1 & 8 & 400 & $3.27 \times 10^{05}$ & 0.050 & 0.023 & 0.040 & $6.54 \times 10^{06}$ & 0.57 & $1.03 \times 10^{07}$ & 0.538 \\
\hline ol & 8 & 600 & $5.28 \times 10^{04}$ & 0.058 & 0.042 & 0.057 & $9.07 \times 10^{05}$ & 0.74 & $1.58 \times 10^{06}$ & 0.386 \\
\hline 02 & 8 & 400 & $3.36 \times 10^{05}$ & 0.054 & 0.095 & 0.032 & $6.26 \times 10^{06}$ & 2.97 & $2.48 \times 10^{07}$ & 0.586 \\
\hline ol & 7 & 600 & $7.25 \times 10^{04}$ & 0.091 & 0.014 & 0.011 & $7.99 \times 10^{05}$ & 1.34 & $1.87 \times 10^{06}$ & 0.112 \\
\hline
\end{tabular}

${ }^{a}$ Accuracies of the fitted parameters are discussed in the section: Reaction transients and their evaluation. 
concentrations and synthetic DNA could not be prepared conveniently yet. As a result of this limitation, the focus was on the second-order step, which is dominant at low concentrations. As shown by the transients for synthetic DNA fragments presented here, the second-order step still is dominant, although contributions from consecutive reactions are also visible.

Kedracka-Krok et al. ${ }^{7}$ did not show transients in the range of low reactant concentrations but only transients at high concentrations in the presence of excess DNA. Obviously the first reaction step is relatively fast under these conditions and probably disappeared to some degree in the dead time period of their instrument. An indication for such a type of problem seems to be the values of their rate constants, which are much lower than those obtained in the present investigation as well as previously by Kleinschmidt et al. ${ }^{6}$ Another reason for differences may be the state of the DNA: As shown above, special care has to be practiced to avoid the presence of hairpin structures in mixtures of the fragments ol_a and o1_b. The data in Figure 1 demonstrate that these hairpins lead to substantial changes of the reaction transients.

Some other potential sources for differences should be discussed: Kedracka-Krok et al. ${ }^{7}$ used a mutant Tet repressor with a single Trp residue in position 43 . A control experiment with a sample of this mutant repressor, generously provided by Kedracka-Krok, revealed transients similar to those obtained with the wt-protein (in standard buffer containing $150 \mathrm{mM}$ $\mathrm{NaCl}$. at $20^{\circ} \mathrm{C}$ at $0.3 \mu \mathrm{M}$ final concentration of both repressor and operator).

Kedracka-Krok et al. ${ }^{7}$ mention in their discussion the possibility that the results of Kleinschmidt et al. ${ }^{6}$ are affected by their use of the natural tandem operator and suggest that there may be some cooperativity of repressor binding. A careful analysis of experimental data showed that cooperativity of repressor binding to the sites of the tandem operator is negligible. ${ }^{25}$ This is confirmed by the results of the present investigation.

Finally, there is a difference in the data analysis with respect to the photoreaction. Kedracka-Krok et al. ${ }^{7}$ do not mention the photoreaction at all, whereas it has been considered both in the evaluations of Kleinschmidt et $\mathrm{al}^{6}{ }^{6}$ and in the present case. Kleinschmidt et al. addressed the photoinduced decrease of the fluorescence intensity by addition of a linear term to the fitted change of the fluorescence intensity. This very simple treatment is one of the reasons why evidence for reaction $\operatorname{step}(\mathrm{s})$ in addition to the bimolecular one was not detected. The present analysis is based on the ratio correction procedure described above, which is an approximation and may not be correct in every detail. In spite of potential problems, we think that the ratio correction procedure is at least better than complete disregard. In view of potential problems, we did not attempt to fit and interpret every minor detail of the transients and restricted the analysis to the main features of the reaction.

The results of the present investigation confirm the very strong dependence of the recognition rate on the salt concentration. This dependence was already found by Kleinschmidt et al. ${ }^{6}$ but in that case was based only on transients measured at a single low concentration of reactants. Now the dependence of the transients on the reactant concentration is an essential basis of the evaluation and thus the rate constants can be accepted as more reliable than previously. The detailed analysis of the fluorescence quenching as a function of the reactant concentration at various salt concentrations demonstrates a clear dependence of fluorescence quenching on the salt concentration. Thus, a basic assumption used previously - both by Kleinschmidt et al. ${ }^{6}$ and by Kedracka-Krok et al. ${ }^{7}$ - for the evaluation of equilibrium constants is not valid. The new values for the equilibrium constants $K$ are clearly lower than presented previously. Moreover, the dependence of $K$-values on the salt concentration indicates $\sim 5$ electrostatic contacts ${ }^{21,22}$ between Tet repressor and the operator - instead of $7-8$ found previously. ${ }^{6}$

Binding constants of Tet repressor to operator DNA were reported in two independent investigations. A methylation protection assay was applied by Lederer et al. ${ }^{26}$ to analyze the induction of Tet repressor by tetracycline. They used binding constants at the high level reported previously by Kleinschmidt et al.j $^{6}$ apparently they did not have sufficiently clear independent experimental information on the binding affinity in the absence of tetracycline. Kamionka et al. ${ }^{27}$ reported a binding constant of $5.6 \pm 2 \times 10^{9} \mathrm{M}^{-1}$ at $150 \mathrm{mM} \mathrm{NaCl}$. Analysis of the Scatchard plot presented in their publication provides a binding constant of $3.6 \times 10^{8} \mathrm{M}^{-1}$, which is close to the value derived from the present stopped flow data.

The simple kinetic model of Kleinschmidt et al. ${ }^{6}$ implying a pre-equilibrium closely corresponding to nonspecific binding followed by a relatively fast conversion to the specific complex cannot be maintained. The experimental transients demonstrate the existence of at least one relatively slow reaction step after the initial bimolecular reaction. Obviously nonspecific binding is expected to be involved in the process, but the approximation used in the previous model was too simple.

The strong dependence of the association rate constant on the salt concentration clearly reflects the crucial role of electrostatics for binding and kinetics. At $50 \mathrm{mM} \mathrm{NaCl}$, the association rate is $2 \times 10^{8} \mathrm{M}^{-1} \mathrm{~s}^{-1}$, which is close to the limit of diffusion control. The decrease of this rate at $600 \mathrm{mM} \mathrm{NaCl}$ by almost four orders of magnitude is remarkable. The effect indicates that the repressor does not find the operator site as easily, when electrostatic interactions are shielded. Formation of contacts between the specific sites of both reactants is facilitated by electrostatics. The facilitation may be described from different points of view. The standard interpretation ${ }^{28}$ is based on a pre-equilibrium complex corresponding to - but not necessarily identical with - nonspecific binding. The preequilibrium complex mainly results from electrostatic interactions. These interactions may also be described in terms of the particularly strong overall dipole moment of the Tet repressor protein: a value of $3.5 \times 10^{-27} \mathrm{Cm}$, corresponding to $1050 \mathrm{D}$ units, was found by measurements of the electric dichroism ${ }^{29}$ and is consistent with the charge distribution found in the crystal structure ${ }^{17}$ of the protein. The positive end of this dipole is in the direction of the recognition $\alpha$-helices and thus is useful for a favorable orientation of the recognition sites toward the negatively charged operator. This type of effect has been described in other cases of reactions as electrostatic steering. ${ }^{30,31}$ Apparently electrostatic steering contributes to the high rate of Tet repressor binding to its operator at reduced salt concentrations but is shielded at high salt.

Molecular dynamics simulations of the tetracycline-induced dissociation of the TetR-operator complex ${ }^{32}$ showed that the allosteric conformation change induced as a first step dissociation of one of the two DNA binding domains. In analogy to this mechanism, TetR-binding to operator DNA may proceed as a two-step reaction with initial binding of only one protein subunit followed by binding of the second one. 
The parameters determined for the two-step reaction from the present experimental data are not consistent with this interpretation because the equilibrium constant observed for the first step is much higher than that expected for binding of a single subunit.

\section{CONCLUSIONS}

The Tet repressor is particularly useful to study the mechanism of specific protein-DNA recognition because its binding to operator DNA is associated with a convenient decrease of protein fluorescence. Although the analysis is facilitated by this technical advantage, there is a striking contrast in the conclusions of two previous investigations. To clear the contradiction, binding of Tet repressor to operator DNA has been reanalyzed by fluorescence stopped flow measurements. Using an operator DNA without complications resulting from a duplex-hairpin transition and a ratio procedure for correction of a photobleaching effect, we have obtained sets of reaction transients for a time range from $0.5 \mathrm{~ms}$ up to $135 \mathrm{~s}$. For given salt concentrations, sets of transients at three different reactant concentrations were combined for global fitting. The data demonstrate a bimolecular reaction followed by at least one intramolecular reaction. The bimolecular association rate constant is $2 \times 10^{8} \mathrm{M}^{-1} \mathrm{~s}^{-1}$ at $50 \mathrm{mM} \mathrm{NaCl}$ and decreases to $5 \times 10^{4} \mathrm{M}^{-1} \mathrm{~s}^{-1}$ at $600 \mathrm{mM} \mathrm{NaCl}$. The remarkably strong decrease of the rate at high salt indicates the substantial influence of electrostatic interactions and may be attributed to initial formation of a pre-equilibrium complex. A contribution from electrostatic steering resulting from the known high dipole moment of Tet repressor is suggested. The rate constants for the intramolecular reaction are in the range of $\sim 0.1 \mathrm{~s}^{-1}$.

Fluorescence quenching is dependent on the salt concentration. The overall binding constant to operator $\mathrm{O} 1$ is $5 \times 10^{8}$ $\mathrm{M}^{-1}$ at $150 \mathrm{mM}$ salt and the salt dependence of this constant indicates a number of $\sim 5$ ion contacts for the specific complex of the TetR dimer.

\section{AUTHOR INFORMATION}

\section{Corresponding Author}

*E-mail: dpoersc@gwdg.de.

\section{Notes}

The authors declare no competing financial interest.

\section{ACKNOWLEDGMENTS}

We are grateful to Stephanie Platzer for preparation of Tet repressor samples and to Dr. Sylwia Kedracka-Krok for a sample of a Tet repressor mutant and for discussions. We also thank Dr. Christoph Kleinschmidt for discussions.

\section{REFERENCES}

(1) Riggs, A. D.; Suzuki, H.; Bourgeois, S. J. Mol. Biol. 1970, 48, 6783.

(2) Riggs, A. D.; Bourgeois, S.; Cohn, M. J. Mol. Biol. 1970, 53, 401417.

(3) Winter, R. B.; Berg, O. G.; von Hippel, P. H. Biochemistry 1981, 20, 6961-6977.

(4) Richter, P. H.; Eigen, M. Biophys. Chem. 1974, 2, 255-263.

(5) Halford, S. E. Biochem. Soc. Trans. 2009, 37, 343-348.

(6) Kleinschmidt, C.; Tovar, K.; Hillen, W.; Porschke, D. Biochemistry 1988, 27, 1094-1104.

(7) Kedracka-Krok, S.; Wasylewski, Z. J. Protein Chem. 1999, 18, $117-125$.
(8) Levy, S. B.; McMurry, L. M.; Barbosa, T. M.; Burdett, V.; Courvalin, P.; Hillen, W.; Roberts, M. C.; Rood, J. I.; Taylor, D. E. Antimicrob. Agents Ch. 1999, 43, 1523-1524.

(9) Ettner, N.; Muller, G.; Berens, C.; Backes, H.; Schnappinger, D.; Schreppel, T.; Pfleiderer, K.; Hillen, W. J. Chromatogr., A 1996, 742, 95-105.

(10) Altschmied, L.; Hillen, W. Nucleic Acids Res. 1984, 12, 21712180 .

(11) Gruber, A. R.; Lorenz, R.; Bernhart, S. H.; Neuboock, R.; Hofacker, I. L. Nucleic Acids Res. 2008, 36, W70-W74.

(12) Tataurov, A. V.; You, Y.; Owczarzy, R. Biophys. Chem. 2008, $133,66-70$.

(13) Owczarzy, R.; Tataurov, A. V.; Wu, Y.; Manthey, J. A.; McQuisten, K. A.; Almabrazi, H. G.; Pedersen, K. F.; Lin, Y.; Garretson, J.; McEntaggart, N. O.; et al. Nucleic Acids Res. 2008, 36, W163-W169.

(14) Porschke, D. Biochemistry 2012, 51, 4028-4034.

(15) Humphrey, W.; Dalke, A.; Schulten, K. J. Mol. Graph Model 1996, 14, 33-38.

(16) Orth, P.; Cordes, F.; Schnappinger, D.; Hillen, W.; Saenger, W.; Hinrichs, W. J. Mol. Biol. 1998, 279, 439-447.

(17) Orth, P.; Schnappinger, D.; Hillen, W.; Saenger, W.; Hinrichs, W. Nat. Struct. Biol. 2000, 7, 215-219.

(18) Tung, C. S.; Carter, E. S. Comput. Appl. Biosci. 1994, 10, 427433

(19) Kuzmic, P. Anal. Biochem. 1996, 237, 260-273.

(20) Kuzmic, P. Methods Enzymol. 2009, 467, 247-280.

(21) Manning, G. S. Q. Rev. Biophys. 1978, 11, 179-246.

(22) Record, M. T.; Anderson, C. F.; Lohman, T. M. Q. Rev. Biophys. 1978, 11, 103-178.

(23) Wissmann, A.; Meier, I.; Wray, L. V.; Geissendorfer, M.; Hillen, W. Nucleic Acids Res. 1986, 14, 4253-4266.

(24) Wissmann, A.; Meier, I.; Hillen, W. J. Mol. Biol. 1988, 202, 397406

(25) Kleinschmidt, C.; Tovar, K.; Hillen, W. Nucleic Acids Res. 1991, $19,1021-1028$

(26) Lederer, T.; Takahashi, M.; Hillen, W. Anal. Biochem. 1995, 232, 190-196.

(27) Kamionka, A.; Bogdanska-Urbaniak, J.; Scholz, O.; Hillen, W. Nucleic Acids Res. 2004, 32, 842-847.

(28) Lohman, T. M. CRC Critical Reviews in Biochemistry 1985, 19, $191-245$.

(29) Porschke, D.; Tovar, K.; Antosiewicz, J. Biochemistry 1988, 27, 4674-4679.

(30) Davis, M. E.; Madura, J. D.; Sines, J.; Luty, B. A.; Allison, S. A.; McCammon, J. A. Methods Enzymol. 1991, 202, 473-497.

(31) Antosiewicz, J.; Wlodek, S. T.; McCammon, J. A. Biopolymers 1996, 39, 85-94.

(32) Aleksandrov, A.; Schuldt, L.; Hinrichs, W.; Simonson, T. J. Mol. Biol. 2008, 378, 898-912. 